\title{
An Experimental String of the NT1000 Baikal Neutrino Telescope
}

A. V. Avrorin ${ }^{a}$, V. M. Aynutdinov ${ }^{a}$, I. A. Belolaptikov $b$, D. Yu. Bogorodsky ${ }^{c}$, N. M. Budnev ${ }^{c}$, R. Wischnewski ${ }^{d}$, O. N. Gaponenko ${ }^{a}$, K. V. Golubkov ${ }^{b}$, O. A. Gress ${ }^{c}$, T. I. Gress ${ }^{c}$, O. G. Grishin ${ }^{c}$, I. A. Danil'chenko ${ }^{a}$, Zh.-A. M. Dzhilkibaev ${ }^{a}$, G. V. Domogatsky ${ }^{a}$, A. A. Doroshenko ${ }^{a}$, A. N. Dyachok ${ }^{c}$, V. A. Zhukov ${ }^{a}$, A. V. Zagorodnikov ${ }^{c}$, A. M. Klabukov ${ }^{a}$, A. I. Klimove ${ }^{e}$ K. V. Konischev ${ }^{b}$, A. V. Korobchenko ${ }^{c}$, A. P. Koshechkin ${ }^{a}$, L. A. Kuzmichev ${ }^{f}$, V. F. Kulepov ${ }^{g}$, D. A. Kuleshov ${ }^{a}$, V. I. Lyashuk ${ }^{a}$, E. Middell ${ }^{d}$, M. B. Milenin ${ }^{g}$, R. R. Mirgazov ${ }^{c}$, S. P. Mikheyev ${ }^{a}$, E. A. Osipova , $^{b}$ A. I. Panfilov ${ }^{a}$, L. V. Pan'kov ${ }^{c}$, G. L. Pan'kov ${ }^{c}$, A. A. Perevalov ${ }^{c}$, D. P. Petukhov ${ }^{a}$, E. N. Pliskovsky ${ }^{b}$, V. A. Poleschuk ${ }^{a}$, E. G. Popova $f$, V. V. Prosinf ${ }^{\prime}$ M. I. Rozanov ${ }^{h}$, V. Yu. Rubtzov ${ }^{c}$, E. V. Ryabov ${ }^{c}$, O. V. Suvorova ${ }^{a}$, B. A. Tarashansky ${ }^{c}$, S. V. Fialkovsky ${ }^{g}$, B. A. Shaibonov ${ }^{b}$, A. A. Sheifler ${ }^{b}$, A. V. Shirokov ${ }^{f}$, Ch. Spiering ${ }^{d}$, and A. S. Yagunov ${ }^{c}$

${ }^{a}$ Institute for Nuclear Research, Russian Academy of Sciences, pr. Shestidesyatiletiya Oktyabrya 7a, Moscow, 117312 Russia

${ }^{b}$ Joint Institute for Nuclear Research, ul. Joliot-Curie 6, Dubna, Moscow oblast, 141980 Russia

c Irkutsk State University, ul. Karla Marksa 1, Irkutsk, 664003 Russia

${ }^{d}$ Das Deutsche Elektronen Synchrotron DESY, Platanenallee 6, Zeuthen, D-15738 Deutschland

${ }^{e}$ Russian Research Centre Kurchatov Institute, pl. Akademika Kurchatova 1, Moscow, 123182 Russia

${ }^{f}$ Skobel'tsyn Institute of Nuclear Physics, Moscow State University, Moscow, 119992 Russia

${ }^{g}$ Nizhni Novgorod State Technical University, ul. Minina 24, Nizhni Novgorod, 603950 Russia

${ }^{h}$ St. Petersburg State Marine Technical University, ul. Lotsmanskaya 3, St. Petersburg, 190008 Russia

Received February 15, 2011

\begin{abstract}
A project of the NT1000 deep-water neutrino telescope with an effective volume of $\sim 1 \mathrm{~km}^{3}$ is currently being developed by the BAIKAL collaboration. The telescope will be located in Lake Baikal in close vicinity of the NT200+ detector, which is currently in operation. The telescope will be composed of 12 clusters with 8 similar strings of optical modules in each (each string has two sections of the NT1000 optical modules). The section of the NT1000 optical modules has been developed using higher-efficiency photomultiplier tubes and state-of-the-art electronics. The field tests of the experimental string consisting of two sections with six optical modules in each have been performed. The results of these investigations are used in the project of the NT1000 neutrino telescope and in the hydrological study of Lake Baikal.
\end{abstract}

DOI: $10.1134 / \mathrm{S} 0020441211040178$

\section{INTRODUCTION}

Large-scale neutrino telescopes in natural media are aimed at solving a wide range of fundamental problems: studying natural fluxes of high-energy muons and neutrinos, searching for hypothetical particles-candidates for the Dark Matter particles, etc. Using these telescopes, it is possible to perform interdisciplinary studies in the field of terrestrial sciences at the qualitatively new and higher level. The successful, more than 10-year-long operation of the first-generation neutrino telescopes - the NT200 Baikal neutrino telescope and the AMANDA setup at the South Pole - has proved the feasibility and efficiency of neutrino detection in natural media, which has made it possible to pass to development and implementation of projects of new-generation setups with a sensitive volume of $\sim 1 \mathrm{~km}^{3}$.

A project of the NT1000 neutrino telescope on Lake Baikal with an effective volume of $\sim 1 \mathrm{~km}^{3}$ is currently being developed [1-3]. This project is based on the experience in designing and long-term operation of the NT200 and NT200+ neutrino telescopes [4-6]. The NT200 setup (Fig. 1) has been operating in Lake Baikal since 1998. The list of physics experiments performed at the NT200 covers the whole range of problems characteristic of deep-water neutrino telescopes [7-13]. The NT200 constitutes a spatial array of 192 optical modules (OMs) located at depths of $1100 \mathrm{~m}$ and more. Hybrid QUASAR-370 photomultiplier tubes [14] with a hemispherical 37-cm-diameter photocathode are used in the OM as light sensors capable 


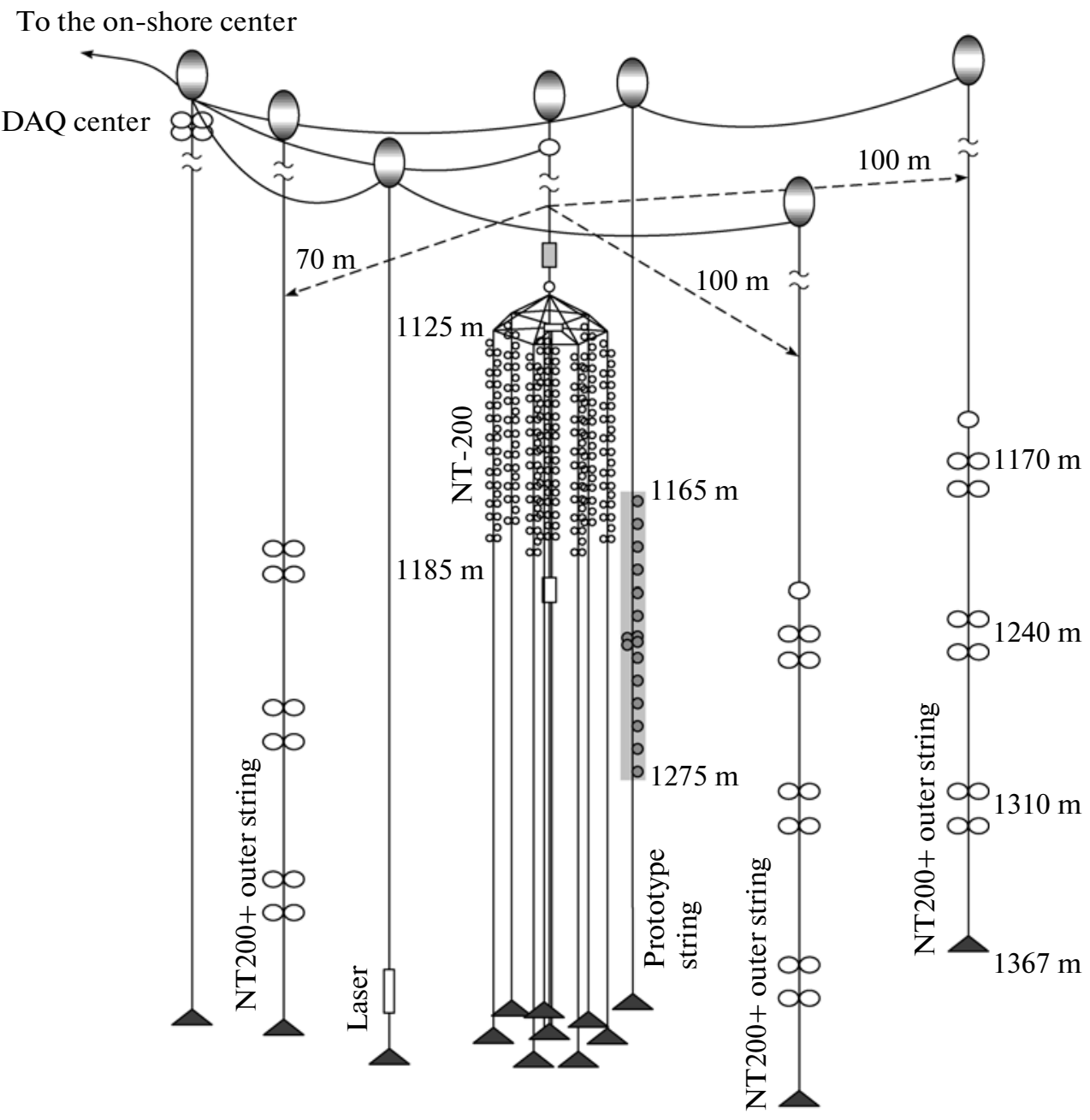

Fig. 1. NT200+ neutrino telescope and the experimental NT1000 string.

of detecting Cherenkov radiation from relativistic muons and cascade showers. Figure 1 also presents three supplementary external strings located at a distance of $100 \mathrm{~m}$ from the NT200 center, which were installed in 2005 (the NT200+ telescope modification). The experience gained in operating the NT200+ has made it possible to investigate the specific features in detection of muons and cascade showers by the rarefied (with respect to the NT200) array of OMs spaced 150-20 m apart. Similar distances between OMs are expected to be used in the NT1000.

The NT1000 neutrino telescope is being designed as a system of similar OM sections. Each section is a self-contained detecting system comprising photodetectors, measuring electronics, and subsystems of control, triggering, and data transmission. The prototype string composed of two sections of this type is presented in Fig. 1. This prototype has been developed for the NT1000 setup and installed in Lake Baikal for long-term field testing. In this paper, we describe the data acquisition system developed for the string of the
NT1000 OMs and discuss the test results for its main components.

\section{OPTICAL MODULE}

Optical modules converting Cherenkov radiation of muons and cascade showers into electrical signals are the detecting elements of each section. The OM consists of the following components: a photomultiplier tube (PMT), a controller, a signal amplifier, a LED-based calibration system, and a high-voltage converter. The block diagram of the OM is shown in Fig. 2.

Selection of the optimal PMT for the NT1000 setup is a separate task. The main requirements specified for the PMT are as follows: the high time resolution (a few nanoseconds) at a large photocathode area and the maximum quantum efficiency at a minimum inherent noise level. These requirements are met to the maximum extent for the R8055 and R7081HQE PMTs (Hamamatsu, Japan) with hemispherical pho- 


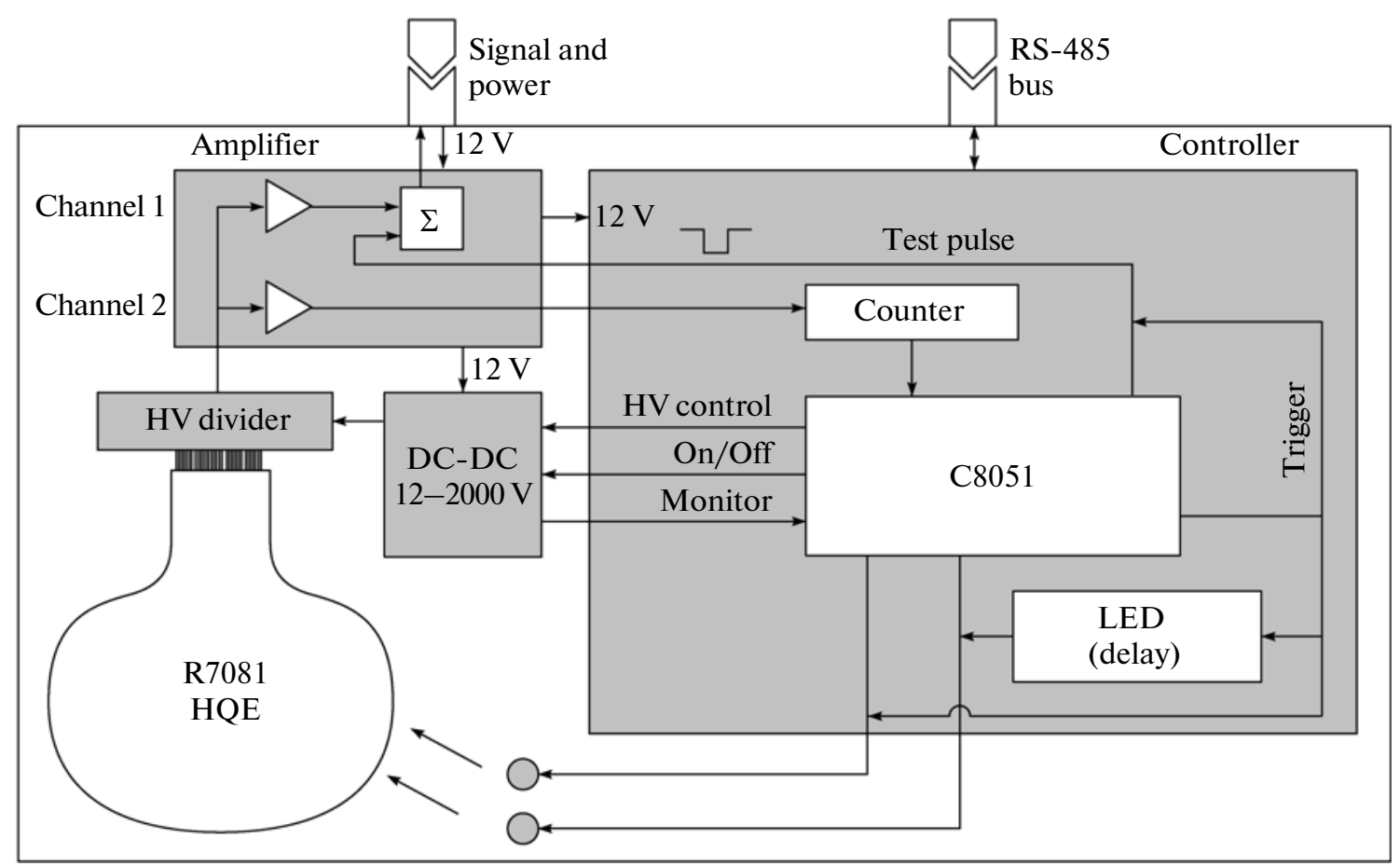

Fig. 2. Block diagram of the OM.

tocathodes, photocathode areas of $\sim 1000$ and $\sim 500 \mathrm{~cm}^{2}$, and quantum efficiencies of $\sim 20$ and $>30 \%$, respectively. Both PMTs are now being tested in Lake Baikal as parts of the NT1000 prototype section.

The high voltage applied to the PMT (1200$1600 \mathrm{~V}$ ) has positive polarity (the photocathode is grounded). It is individually selected for each PMT so that the gain of the PMT dynode system is $\sim 10^{7}$. To maintain reliable operation of spectrometric channels in the telescope, the PMT signal is additionally amplified by a factor of 10 , which ensures both the high mean value of the single-photoelectron signal from the PMT $(30-40 \mathrm{mV})$ relative to the mean noise amplitude in the spectrometric channel, and the required linearity range of the channel response [up to $\sim 100$ photoelectrons (PE)]. The amplifier and the PMT voltage divider are mounted on a common board. The first channel of the amplifier is used in spectrometric measurements, and the second amplifies the signals arriving at the counter of PMT noise pulses.

Operation of the $\mathrm{OM}$ is controlled over a deepwater RS-485 bus via a special controller developed for the NT1000 on the basis of the C8051F124 processor. The main functions executed by the controller are as follows:

— controlling the high voltage applied to the PMT;

- performing amplitude and time calibrations of the spectrometric channels by LED-based pulsers; and

INSTRUMENTS AND EXPERIMENTAL TECHNIQUES
- monitoring the main parameters of the PMT and the OM electronics during long-term exposure.

The electrical power is supplied for the PMT via the voltage divider with a total resistance of $18 \mathrm{M} \Omega$ by the PHV12-2.0K2500P high-voltage DC/DC converter (TRACO POWER), the output voltage of which can be varied over the range of $0-2.5 \mathrm{kV}$. The control input of the DC/DC converter is used to set the operating value of the MT voltage. The control voltage is produced by a digital-to-analog converter (DAC) of the controller in the range of $0-2.5 \mathrm{~V}$ with a step of $1 \mathrm{mV}$; this ensures the accuracy in setting the PMT voltage of $1 \mathrm{~V}$ or better.

Two L7113 PBC-A LEDs (Kingbright) are used for the amplitude and time calibration of the PMT. The maximum of the LED emission spectrum corresponds to a wavelength of $445 \mathrm{~nm}$, and the duration of the light pulse is $\sim 5 \mathrm{~ns}$. The controller executes precision control over the emission intensity of the LEDs and regulates the delay between their pulses. The intensity can be regulated in the range of $0-10^{8}$ photons/pulse, and the range of delay times is $0-1000 \mathrm{~ns}$ (the step is $\sim 100 \mathrm{~ns}$, and the error of delay time setting is $\sim 1 \mathrm{~ns}$ ). The crosstalk between two LED channels does not exceed $0.5 \%$ if their light signal amplitudes are equal. The light pulses from the LEDs are transmitted to the PMTs over fiber-optic cables $\sim 0.5 \mathrm{~m}$ long.

Control of the PMTs and OM electronics implies regular monitoring of their main parameters and operating conditions. The controllable parameters of the

Vol. $54 \quad$ No. $5 \quad 2011$ 


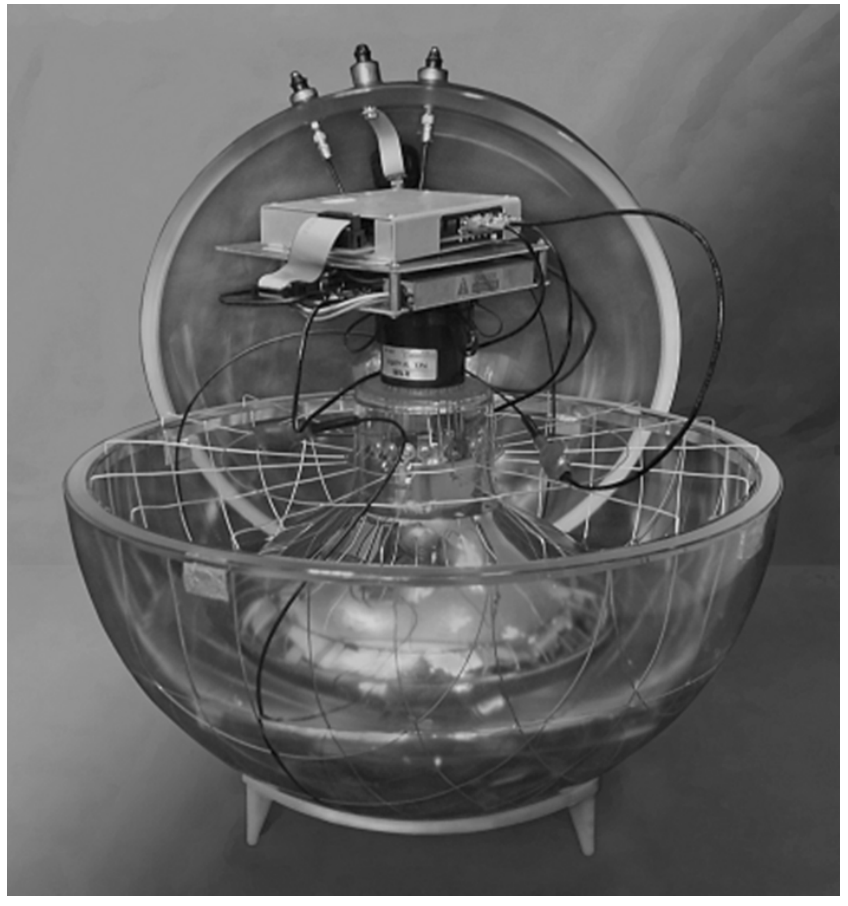

Fig. 3. Optical module based on the R7081HQE PMT.

PMTs are the high voltage, the delay time, and their inherent noise level. The monitor output, the voltage at which is proportional to the total voltage across the voltage divider, is used to control the high voltage in the PMT's voltage divider. This voltage is measured by the analog-to-digital converter (ADC) of the $\mathrm{OM}$ controller.

The technique for monitoring the PMT delay time is based on measuring the time between the triggering signal applied to the LED and the LED signal detected by the PMT. In the mode of delay time measurements, the signal coincident in time with the triggering signal is applied to the amplifier output stage in the OM spectrometric channel. Two signals are produced thereby; the time interval between them is determined by the PMT delay time and is measured in the central module of the section (see Section 3) with an accuracy of $\sim 2$ ns. It should be noted that the possibility of coercive pulse forming at the OM output is also used to monitor the operability of the section as a unit without the high-voltage being applied to the PMTs.

The inherent noise of the PMTs is measured using the second amplifier channel (its gain is $\sim 20$ ), the signal from which is applied to the nanosecond pulse counter of the OM controller (Fig. 2). The duration of the time interval for counting noise pulses and the interval between measurements can be selected in the range from a few milliseconds to $10 \mathrm{~s}$, depending on the PMT operating conditions. It is possible to adjust the detection threshold of the counter. The minimum operating threshold is limited by the noise pickup amplitude from the processor of the OM controller; its value is $\sim 10 \mathrm{mV}(\sim 0.3 \mathrm{PE})$.

To monitor operation of the OM electronics, the controller provides a means for measuring the temperature and the voltage levels of the OM power supply. A common cable is used to feed the $12-\mathrm{V}$ supply voltage and the PMT signals to the OM. Integrating the power supply with the spectrometric channel simplifies substantially the system of deep-water cable lines of the NT1000 section. However, this approach leads to an increase in the spectrometric channel noise to a level of 5-7 $\mathrm{mV}$ due to the inherent noise of the power source. The power consumption of the $\mathrm{OM}$ is $200 \mathrm{~mA}$ $\times 12 \mathrm{~V}$.

The external appearance of the OM with the R7081HQE PMT is shown in Fig. 3. The electronic components of the $\mathrm{OM}$ are enclosed in a deep-water VITROVEX glass sphere $42 \mathrm{~cm}$ in diameter, which consists of two hemispheres. To reliably fix the hemispheres in place after the $O M$ is assembled, air is pumped out of it to a pressure of $\sim 0.7 \mathrm{~atm}$.

The PMT is glued into one of the hemispheres using a silicone RTV adhesive, which maintains optical contact between the glasses of the sphere and the PMT. To reduce the effect of the Earth's magnetic field, the PMT is shielded with a Permalloy grid. The controller, the amplifier, and the high-voltage converter are fixed in place on the PMT base. The drivers with the LEDs are proofed against light and connected to the OM controller through high-voltage SMA connectors. Light emitted by the LEDs is transmitted to the center of the PMT photocathode over optical fibers. When amplified, the PMT signal is outputted from the OM through a deep-water CP-50-862/863 coaxial connector. The power is supplied for the OM through this connector. A similar connector is used in the OM to connect the RS-485 control bus. There is a vacuum valve between these two connectors, to which a vacuum gauge is attached to monitor the pressure inside the OM.

\section{NT1000 SECTION}

The NT1000 section is the basic detecting unit of the setup. Sections are mounted on vertical strings, which are combined in clusters. The basic configuration of the cluster consists of eight strings with two sections in each.

The block diagram of the section is shown in Fig. 4. Each section comprises $12 \mathrm{OMs}$, central module $C M$, and service module $S M$.

The central module combines the functions of data acquisition and control of the section's performance. The analog signals from 12 OMs arrive at the central module over coaxial cables $\sim 100 \mathrm{~m}$ long. The PMT signals are converted into a digital code by three boards of 4-channel 12-bit ADCs with a sampling fre- 
quency of $200 \mathrm{MHz}$ in a time window of $15 \mu$ s. Time lines of events are formed thereby in each channel. Analysis of these lines allows both the amplitude and the detection time of the OM signals to be determined. There are two ring buffers in each ADC channel to record information from the time lines, which minimizes the dead time of the channels.

Apart from the conversion of the analog signals and the intermediate data storage, the ADC boards are used to generate request signals for the channels. The Request signal is produced if the amplitude of the input signal exceeds the predetermined value (in this case, the counts from the four adjacent ADCs on the recorded time line are averaged). To do this, two digital comparators operate in each ADC channel; their thresholds are controlled by the program with a step of $\sim 1.4 \mathrm{mV}$ (0.05 PE). Two types of the Request signals are generated in each ADC channel: Low Threshold ( $\sim 0.3 \mathrm{PE})$ and High Threshold ( 3 PE).

The Request signals from all the ADC channels are fed to the Master board, which produces the Section Request signal. Coincidences of several Low Threshold signals (the majority coincidence circuit) or the coincidences of the Low Threshold and High Threshold signals from any two channels of adjacent OMs are the conditions for generation of the Section Request signal. The information on the combinations of signals, coincidence of which in a predetermined time window results in generating the Section Request signal, is dynamically loaded into the memory of the Master board (the coincidence matrix). The Section Request signals are transmitted over $\sim 1200$-m-long coaxial cables to the cluster center, in which the Acknowledgment signal is generated. This signal is the global trigger for all sections, which maintains their synchronous operation. By this signal, the data from all ADC channels are read out and transmitted to the cluster's data acquisition center, which communicates with the onshore center via a fiber-optic line.

Data are read out of the section over the Ethernet channel of the Master board, which is extended to the required length of $1200 \mathrm{~m}$ via the DSL modem (the data transmission rate is as high as $9 \mathrm{Mbit} / \mathrm{s}$ ). A local underwater RS-485 data bus based on the ASCII protocol is used to set the OM operation conditions, calibrate, and monitor the equipment, for which high transmission rates of commands and data are not needed.

The supply voltage is delivered to the OMs from the linear DC/DC 300-12 V voltage converters located in the service module of the section, over the board combining the power supply and the OM signals. This board also performs relay control of the OM turn-on; it allows OM disconnection from the voltage source in case of a short-circuit failure.

Along with the voltage converters, components of the monitoring and calibration system and an elec- tronic unit of the hydroacoustic positioning system HAPS are also located in the service module. The monitoring system provides information on the supply voltages of the section and each OM (300 and $12 \mathrm{~V})$, the temperature inside the OM, the high voltages applied to PMTs, and the counting rate of PMT background pulses (the counting rates are determined by the dark currents of the PMTs and the emission intensity of the Baikal water). Calibration of the section is performed using two LED-based pulsers, the light pulses from which are distributed among all OM sections over optical cables.

The central and service modules of the section are similar in design to the OM. The electronic circuits of the modules are enclosed in the deep-water VITROVEX glass spheres. A crate with the electronics is mounted on the aluminum ring glued into the bottom hemisphere. Deep-water CP50-862/863 coaxial connectors are used to transmit signals and data. A carrier cable with three power supply wires, one twisted pair, and two coaxial cables is used to supply power $(300 \mathrm{~V})$, transmit data (Ethernet), and synchronize operation (signals Request and Acknowledgment).

\section{CALIBRATION OF THE NT1000 SECTION}

The NT1000 setup has been designed for long-term detection of cosmic ray fluxes. The characteristics of the detection channels may vary over time due to variations in the parameters of the PMTs, the measuring electronic system, and the operating conditions (in particular, the voltage applied to the PMTs). To determine the parameters needed for converting the measured values (the PMT responses) into physical quantities (the photon flux and the photon detection time), time and amplitude calibrations are performed. The first of these is used to determine the intrinsic delay times in channels (the time shifts), which are formed by delays in the PMTs, electronics, and cable lines. The second is used to determine the signal amplitudeto-photoelectrons conversion function. To change from photoelectrons to the Cherenkov photon flux, the quantum sensitivity of the PMT photocathode must be known. The quantum sensitivity is calibrated by an underwater laser-based light source and by atmospheric muon flux.

Time calibration is performed using a LED-based pulser located in the service module. The light pulse from the source follows distinct paths and is delivered to each OM over fiber-optic cables with a calibrated time delay. Taking into account the known time delay in the optical cable, the relative time shifts of each channel are determined from the measured detection times of this pulse. This technique has been tested in operation of the NT200 and NT200+ detectors with a time calibration error of $\sim 2 \mathrm{~ns}$. The drawback of this approach is the necessity to mount 12 fiber-optic 




Fig. 4. Functional diagram of the NT1000 and its arrangement on the string: $(C M)$ central module, $(S M)$ service module, (HAPS) hydroacoustic positioning system, (LED) light-emitting diode.

cables on a string, which increases considerably the time of its deployment from the ice on Lake Baikal.

The inclusion of the ADC in the measuring channels of the section provides a means for using an alternative time calibration technique based on direct measurements of the signal delay time in the OM (the measuring procedure was presented in Section 2). To obtain the time shifts of the channels, one can easily sum the signal delay time in the OM and the measured laboratory value of the delay time in the signal cables (this procedure is equivalent to calibration of the delays in fiber-optic cables). The arrangement of the electronic system in the NT200 measuring channel has made it impossible to apply this approach to the time calibration; therefore, both approaches were used in the field tests of the NT1000 section (see Section 5).

The amplitude calibration of the spectrometric channels is based on a widespread technique of measuring single-photoelectron spectra from a PMT using a LED-based calibration system (Section 2). The specific feature of applying this method to the NT1000 consists in using two signals from two OM LEDs to measure the single-photoelectron spectrum. The single-photoelectron signals due to the first LED are recorded only in the presence of the signal from the second LED with an amplitude of $\sim 100 \mathrm{PE}$ and a delay time of $\sim 100 \mathrm{~ns}$. Such an approach allows single-photoelectron signals to be reliably discriminated from the
PMT noise. An example of the calibration single-photoelectron spectrum from the PMT is shown in Fig. 5. The mean charge of the single-photoelectron signal, determined from this spectrum, corresponds to ADC channel 78 (parameter $P_{3}$ in Fig. 5) with allowance for the pedestal value in the channel (parameter $P_{1}$ ).

The conversion coefficient of the spectrometric channel obtained thereby can be used only in the linear conversion region limited by the signals of $\sim 100$ PE. It is this region that is of particular interest in reconstruction of physical events in the NT1000. Large signals exceed the limits of the ADC dynamic range and are nonlinearly converted. For the amplitude information in the region of nonlinear conversion of the channel to be used, it is necessary that its calibration characteristic be measured. The calibration characteristic of the channel is measured by a system of two OM LEDs by sequentially doubling the luminous flux and recording the respective OM response.

An example of the calibration curve of the channel is shown in Fig. 6. When the calibration characteristic is measured by this method, errors are accumulated as the signal amplitude increases. The systematic shift in the region of up to $\sim 103 \mathrm{PE}$, measured by the independent method, did not exceed $10 \%$, which corresponded to the statistical error of measurements at the boundary of the linear range. 


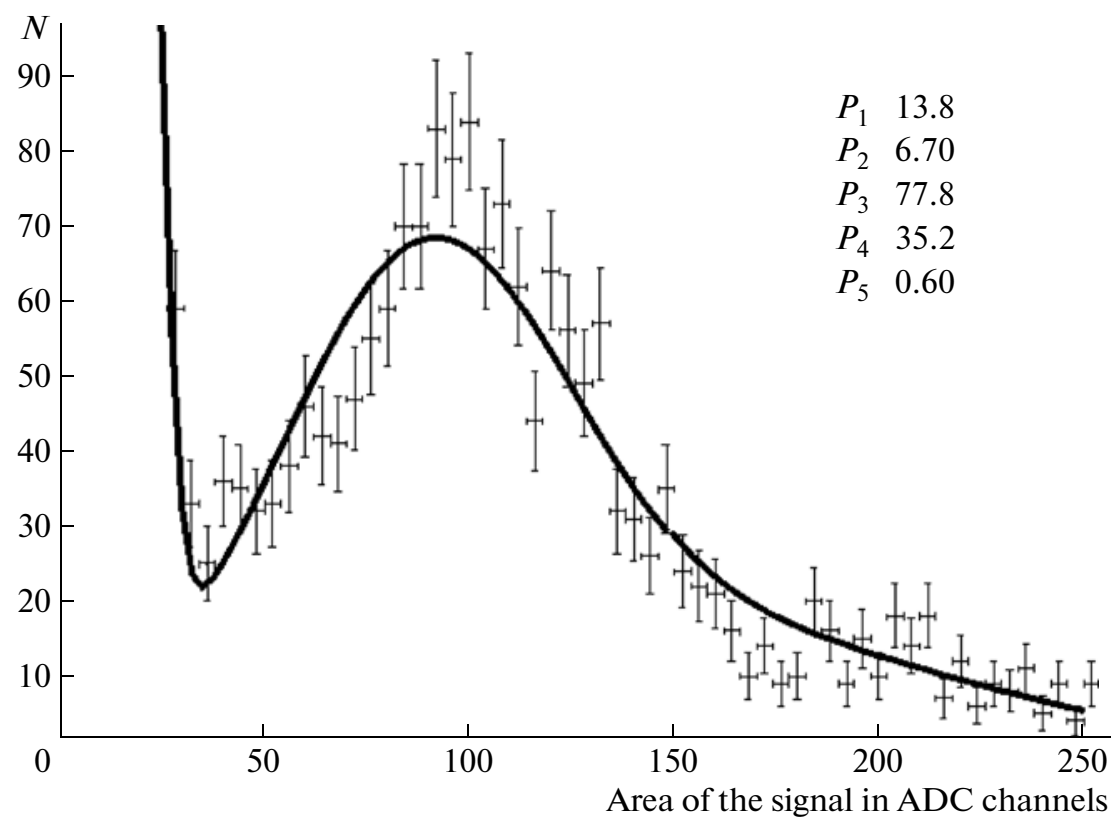

Fig. 5. Example of the amplitude calibration by the single-photoelectron PMT spectrum.

\section{FIELD TESTS OF THE NT1000 SECTION IN LAKE BAIKAL}

The field tests of the equipment of the NT1000 section were performed in Lake Baikal in 2008-2010. In 2008, a string composed of six OMs was installed with the aim of investigating the technique of Cherenkov radiation detection by ADC-based electronics. In 2009, the string was extended to 12 OMs combined in two sections with six OMs in each (Fig. 7). The separations between the OMs were $10 \mathrm{~m}$, and the total string length was $110 \mathrm{~m}$. All PMTs of the string, except for the two bottom PMTs, were oriented so that their photocathodes looked upward. There are two central service and computer ( $P C$ sphere) modules at the string center, which act as the data acquisition center for the NT1000 cluster. The XP1807 PMTs with a 12" photocathode (Photonis) and the R8055 PMTs with a 13" photocathode (Hamamatsu) were used in the string. In 2009, Photonis stopped manufacturing PMTs with a 12" photocathode, and Hamamatsu announced a new R7081HQE PMT with a 10" photocathode featuring an increased quantum efficiency $(>30 \%)$. The XP1807 PMTs were replaced with R7081HQE in April of 2010, and works with the experimental string were continued until August of 2010.

The control and data transmission system of the string is made as close as possible to the base configuration developed for the NT1000 section (Fig. 4). The task of the cluster's data acquisition center is performed by a computer module providing generation of common signal Acknowledgment for two sections and data transmission to the on-shore center over the
Ethernet channel via the DSL modem. Since the computer module is located in close proximity to the central modules of the string, it communicates with the $C M_{1}$ and $C M_{2}$ without recourse to the DSL modems, as is the case of the base configuration of the electronics in the section. The electronics of the hydroacoustic positioning system HAPS is housed in its own modules at the top and bottom of the strings.

The main task performed with the experimental string was to comprehensively test operation of all electronic units, deep-water cable lines, and bearing constructions under conditions of long-term exposure to cosmic rays. In 2008-2010, the experimental string in different configurations was in operation for a total of $\sim 12$ months. In this period, no significant operational errors or seal failures have been detected in the basic string units (the OMs, ADCs, control units,

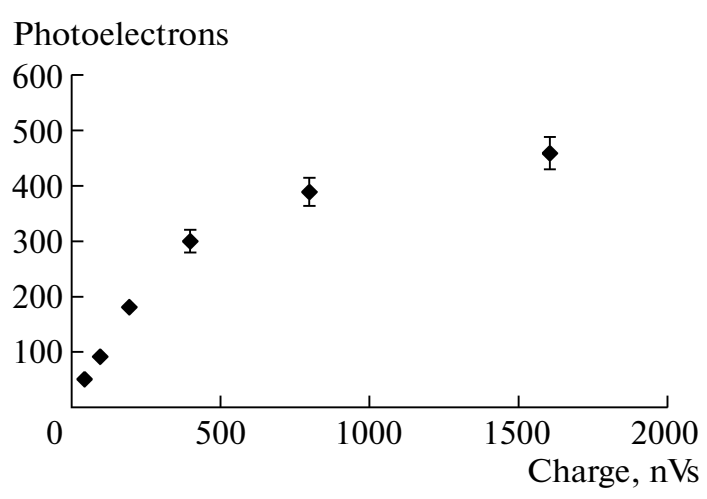

Fig. 6. Example of the calibration characteristic of the spectrometric channel. 

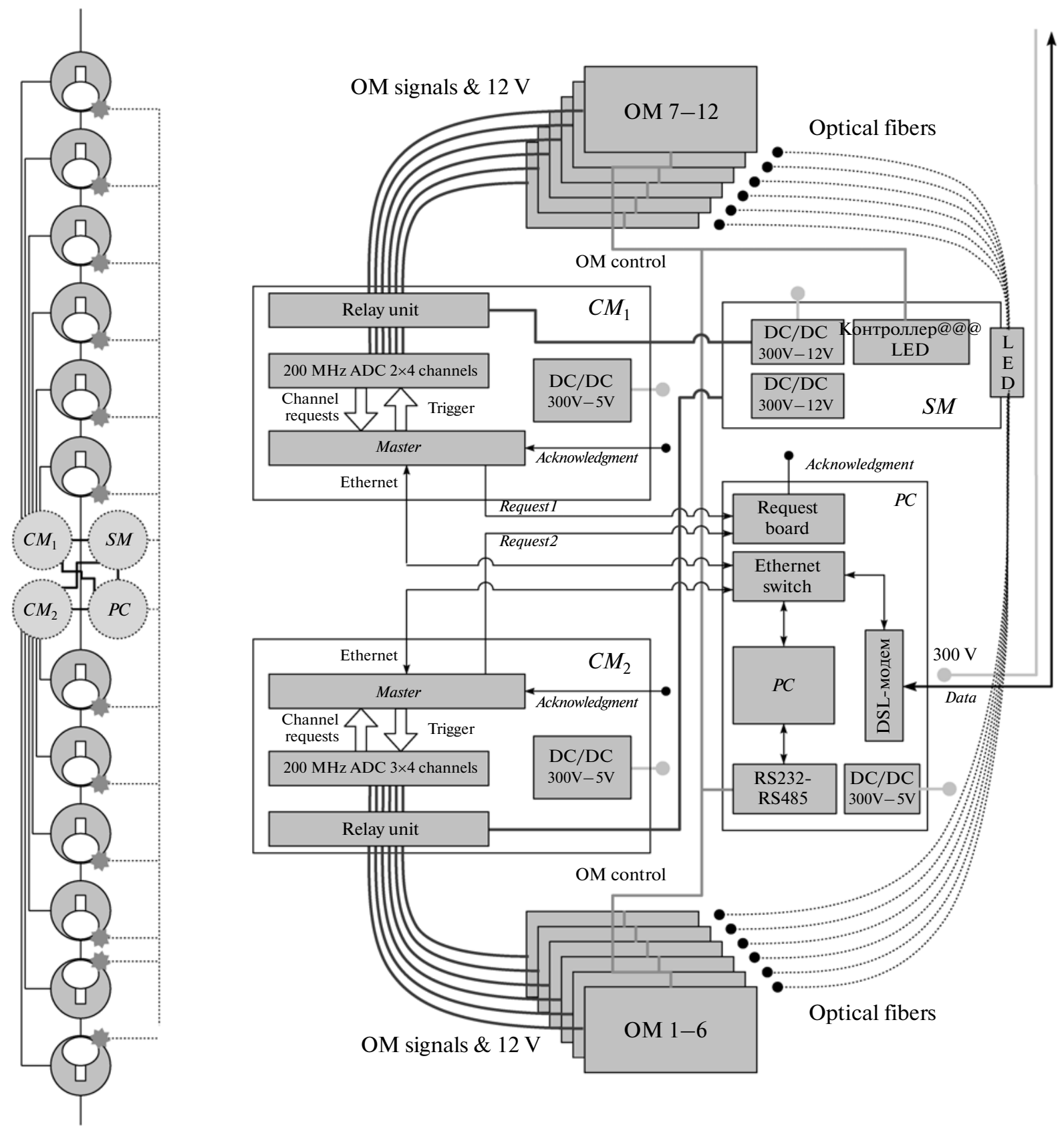

Fig. 7. Block diagram and arrangement of modules in the experimental string composed of two sections with six OMs in each: $(C M)$ central module, $(S M)$ service module, $(P C)$ computer module, and (LED) light-emitting diode.

deep-water cables, and connectors). The outages in the string operation were caused by failures of the DC/DC converters of the service module. Based on this experience, a lightning guard system and a backup power supply of the string have been developed and introduced into the setup.

The key parameter of neutrino telescopes is their angular resolution, which must be substantially better than $1^{\circ}$. The angular resolution depends, primarily, on the accuracy in measuring the time of Cherenkov radiation detection by each detector channel, which is determined by the two parameters of the detecting system: the time resolution of the channels and the accuracy of their time calibration. These parameters of the experimental string were investigated in the modes of detection of light pulses from the LEDs, the calibration laser source, and atmospheric muons.

The time resolution of the channels was measured in the mode of string calibration by the LED-based light pulser. The pulser located at the center of the string inside the service module generated a set of double pulses with a rigidly fixed time interval (delay) 


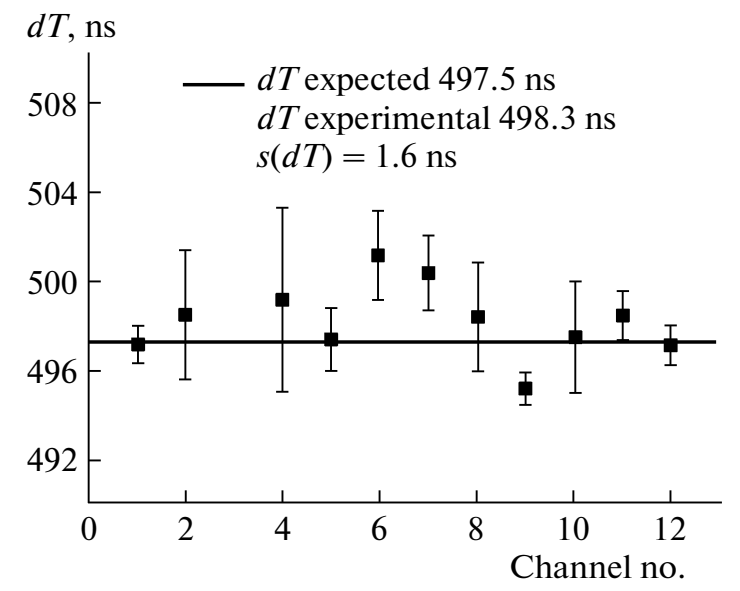

Fig. 8. Determination of the delay time between the signals.

between them. The delay time ( $\sim 500 \mathrm{~ns})$ was selected such that both pulses corresponded to the time window of a single event. The light pulses were transmitted to all OMs in the string over optical cables. The position of the pulses on time lines of the channels was determined by an excess of the fixed threshold, which was selected to be $\sim 0.5 \mathrm{PE}$. The delays between the LED pulses, measured for all string channels, are presented in Fig. 8. The delay time averaged over all channels (498.3 ns) differs from the expected value (497.5 ns) by $\leq 1 \mathrm{~ns}$. The rms deviation of delays averaged over all channels is $1.6 \mathrm{~ns}$. This value characterizes the time resolution of the string channel. The time resolution can be improved, using fits of pulses to determine the positions of the pulses on the time line. The large spread in the rms deviation is explained by the difference in amplitudes of light pulses (from 1 to $100 \mathrm{PE}$ ), which can be attributed to different focusing conditions for light from the LEDs at the entries of optical cables. For this reason, in particular, information on the third channel is absent in Fig. 8.

The accuracy of time calibration of the channels is the second factor affecting the timing characteristics of the detector. Calibration implies determination of relative time shifts of channels $t_{\text {shift }}$, caused by the difference in the delay time of the cable lines in the channels and the PMTs. The time calibration technique was presented in Section 4. The values of calibration parameters $t_{\text {shift }}$, obtained using two methods (by measuring the detection times of the signal common for all channels from the LED-based light pulser and by measuring the intrinsic delay times of the PMTs), coincide with an accuracy of 3 ns, which is greater than the expected value of 2 ns. For subsequent analysis, we selected results of the LED-based time calibration.

The accuracy in measuring the time of Cherenkov radiation detection by the channels of the experimen-

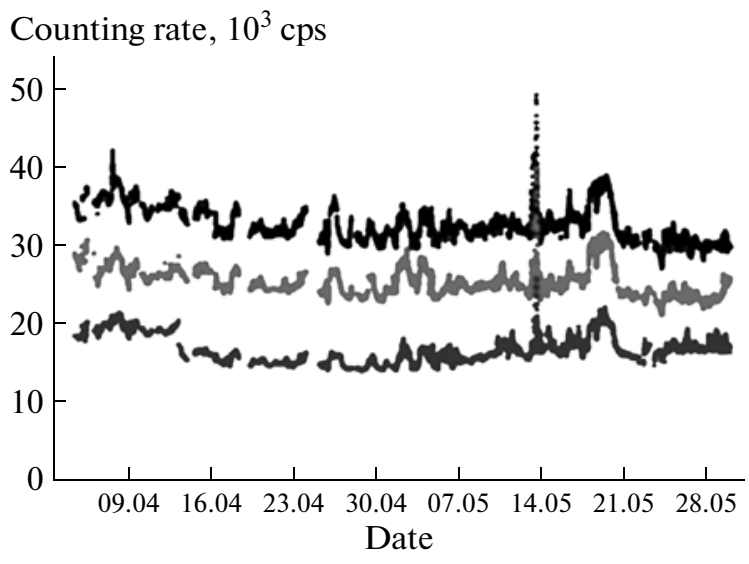

Fig. 9. Counting rate in the three channels of the experimental string over 2-month exposure.

tal string was estimated by the results of its calibration using the calibration laser. The calibration laser was an isotropic light source with an emission intensity as high as $5 \times 10^{13}$ photons/pulse at a wavelength of $475 \mathrm{~nm}$ and a light pulse duration of $<1 \mathrm{~ns}$. The laser was located at a distance of $\sim 100 \mathrm{~m}$ from the experimental string at a depth of $\sim 1.2 \mathrm{~km}$. The hydroacoustic positioning system is capable of determining the mutual position of the laser and the OMs of the string with an uncertainty of $0.2 \mathrm{~m}$. This allows us to compare the expected time of radiation detection by the string channels to the values obtained in the experiment. As the experimentally measured parameter, we used the difference in the triggering time of the channels $d T$. Below, we present the results of comparison of measured values $d T_{\text {meas }}$ to the expected values based on the geometrical considerations $d T_{\text {geom }}$, as functions of the distance between the channels $d R$.

$\begin{array}{cc}d R, \mathrm{~m} & d T_{\text {meas }}-d T_{\text {geom }}, \mathrm{ns} \\ 10 & 0.3 \\ 20 & -1.2 \\ 30 & -2.2 \\ 40 & 0.5 \\ 50 & 1.3\end{array}$

The $d T_{\text {meas }}$ values were obtained by averaging over all combinations of channels located at fixed distance $R$. These results suggest that the accuracy in measuring the detection times by the string channels is $3 \mathrm{~ns}$ or better, which guarantees the required angular resolution of the NT1000 detector.

Analysis of the background conditions for the experimental string in Lake Baikal has not revealed any new effects in comparison with the effects investigated earlier with the NT200 detector. The time dependence of the counting rate of noise pulses (the 


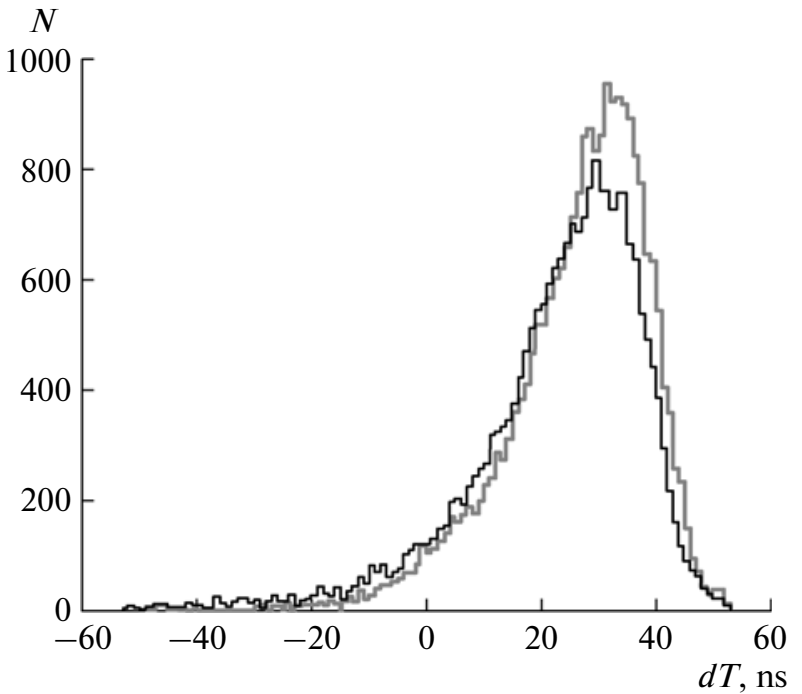

Fig. 10. Distribution in the difference in the triggering time of the string channels comprising the upward-looking PMTs: experiment and simulation of the string response to atmospheric muons.

threshold was $\sim 0.5 \mathrm{PE}$ ) is presented in Fig. 9 for all three string channels spaced $10 \mathrm{~m}$ apart.

The clearly discernible correlation of the counting rates in the channels shows that the major portion of the PMT noise can be attributed to luminescence of the water in Lake Baikal. The experimental results show that the nature of light emission is associated with chemiluminescence processes. The luminescence at large depths is rather stable for most part of the year; nevertheless, there are periods when its intensity grows in value, during which the counting rate of noise pulses increases twofold and more. Apparently, these bursts are due to the features of hydrophysical and hydrobiological processes in Lake Baikal.

Based on the analysis of data on the atmospheric muon flux, the timing characteristics of the experimental string were investigated under conditions approaching the actual operating mode. Figure 10 presents the experimental muon distribution according to the zenith angle, plotted as a dependence on the difference in triggering time $d T$ of the OM pair with the upward-looking PMTs, in comparison with the results of simulation of the string response to atmospheric muons. The experimental distribution is in good agreement with the calculation. The shift of the distributions on the time scale with respect to each other is $\sim 2-3$ ns. This value characterizes the timing accuracy of the experimental string as a unit, including all sources of time measurement errors. The detection process and the obtained characteristics will be similar to those of the downward-looking PMTs in detection of events under investigation due to neutrinos from the bottom hemisphere.

\section{CONCLUSIONS}

A project of the NT1000 deep-water neutrino telescope with an effective volume of $\sim 1 \mathrm{~km}^{3}$ is currently being developed by the BAIKAL collaboration. The telescope will be located in Lake Baikal in close vicinity of the operating NT200+ detector and will be composed of similar units-OM sections specially developed for the NT1000 using state-of-the-art electronic components and computer programs. To test the main elements of the NT1000 section (the optical modules and the ADC-based systems of data acquisition and processing, the system of deep-water cable lines, and the timing parameters of the measuring system), the investigations were carried out with the NT1000 experimental string, which was installed in Lake Baikal and operated jointly with the NT200+ setup. In the period from April of 2008 to August of 2010, the experimental string in different configurations was in operation for a total of $\sim 12$ months. In this period, no significant operational errors or seal failures have been detected in the basic string units (the optical modules, ADCs, control units, deep-water cables, and connectors).

The investigations of the string's timing characteristics, performed using the LED-based light pulser and the calibration laser and during exposure to an atmospheric muon flux, have made it possible to estimate the accuracy in measuring the time of Cherenkov radiation detection by the string channels; this accuracy appeared to be $2-3 \mathrm{~ns}$. The results of these investigations have been used as a basis for the project of the NT1000 neutrino telescope, the termination of which is scheduled for 2011.

\section{ACKNOWLEDGMENTS}

This work was supported by the Ministry for Science and Education of the Russian Federation (project nos. GK 02.740.11.0018, GK 02.518.11.7158, P1242， P2504， P133， P878， P1146， RNP 2.2.1.1/1483, RNP 2.1.1/1539, RNP 2.2.1.1/5901, and the Baikal Research and Educational Center), the Ministry for Science and Education of Germany, and the Russian Foundation for Basic Research (grant nos. 08-02-00432, 08-02-00198, 10-02-10000, 09-0200623, 09-02-12295, and 10-02-10007).

\section{REFERENCES}

1. Aynutdinov, V., Avrorin, A., Balkanov, V., et al., Nucl. Instrum. Meth. Phys. Res., Sect. A., 2009, vol. 602, p. 227.

2. Avrorin, A., Aynutdinov, V., Balkanov, V., et al., Nucl. Instrum. Meth. Phys. Res., Sect. A, 2010. doi:10.1016/j.nima.2010.06.209

3. Avrorin, A., Aynutdinov, V., Balkanov, V., et al., Nucl. Instrum. Meth. Phys. Res., Sect. A, 2011. doi:10.1016/j.nima.2010.09.137 
4. Belolaptikov, I., Bezrukov, L., Borisovets, B., et al., Astropart. Phys., 1997, vol. 7, p. 263.

5. Aynutdinov, V., Avrorin, A., Balkanov, V., et al., Nucl. Instrum. Meth. Phys. Res., Sect. A, 2008, vol. 588, p. 99.

6. Aynutdinov, V., Balkanov, V., Belolaptikov, I., et al., Nucl. Instrum. Meth. Phys. Res., Sect. A, 2006, vol. 567, p. 433.

7. Avrorin, A., Aynutdinov, V., Balkanov, V., et al., Proc. XXXI ICRC, Lodz, Poland. 2009. arXiv:0909.5562.

8. Aynutdinov, V., Avrorin, A., Balkanov, V., et al., Nucl. Instrum. Meth. Phys. Res., Sect. A, 2009, vol. 602, p. 14.

9. Aynutdinov, V., Balkanov, V., Belolaptikov, I., et al., Astropart. Phys., 2006, vol. 25, p. 140.
10. Avrorin, A., Aynutdinov, V., Balkanov, V., et al., Astronomy Let., 2009, vol. 35, p. 651.

11. Aynutdinov, V., Balkanov, V., Belolaptikov, I., et al., Nucl. Instrum. Meth. Phys. Res., Sect. A, 2006, vol. 567, p. 433.

12. Antipin, K., Aynutdinov, V., Balkanov, V., et al., Proc. $X X X$ ICRC, Merida, Mexico. 2007. arXiv:0710.3063.

13. Avrorin, A., Aynutdinov, V., Balkanov, V., et al., Proc. XXXI ICRC, Lodz, Poland. 2009. arXiv:0910.4327.

14. Bagduev, R., Bezrukov, L., Borisovets, B., et al., Nucl. Instrum. Meth. Phys. Res., Sect. A, 1999, vol. 420, p. 138. 